Research Paper

\title{
Finite Element Analysis of Thin Circular Cylindrical Shells
}

\author{
ARUNA RAWAT ${ }^{1, *}$, VASANT MATSAGAR ${ }^{2}$ and A K NAGPAL ${ }^{3}$ \\ ${ }^{1}$ PhD Research Scholar, Department of Civil Engineering, Indian Institute of Technology (IIT) Delhi, \\ Hauz Khas, New Delhi 110 016, India \\ ${ }^{2}$ Associate Professor, Department of Civil Engineering, Indian Institute of Technology (IIT) Delhi, Hauz \\ Khas, New Delhi 110 016, India \\ ${ }^{3}$ Emeritus Professor, Department of Civil Engineering, Indian Institute of Technology (IIT) Delhi, Hauz \\ Khas, New Delhi 110 016, India
}

(Received on 01 May 2016; Accepted on 03 May 2016)

\begin{abstract}
Free vibration finite element (FE) analysis of thin circular cylindrical shells is investigated. The circular cylindrical shell can vibrate in different modes and theoretically infinite modes are possible. The axial mode, $m$ and circumferential mode, $n$, in any of their combinations define the modes and the corresponding modal frequencies. The shell elements are used to model the thin circular cylindrical shells. The eigenvalues of the shell are extracted using block Lanczos iteration method. Detailed mesh convergence studies are performed for different height or length to radius $(H / R)$ ratios. Importantly, selection of appropriate FE mesh size criteria based on the perimeter and height of the circular cylindrical shell, as well as thickness to radius $(h / R)$ ratio are shown for various boundary conditions. The modal frequencies of the cylindrical shell are investigated for different boundary conditions such as clamped-clamped (C-C), clamped-free (C-F), and simply-supported - simplysupported (S-S). The effects of height to radius $(H / R)$ ratio and thickness to radius $(h / R)$ ratio on the modal frequencies of the cylindrical shells are also studied. For all the considered boundary conditions, the modal frequencies of the cylindrical shells increase with higher circumferential mode number and also with the increase in the $h / R$ ratio. The modal frequencies are observed to be the lowest in the case of the C-F boundary condition of the shell.
\end{abstract}

Keywords: Boundary Condition; Circular Cylinder; Finite Element; Modal Frequency; Shell

\section{Introduction}

Thin shells as structural elements are the most predominantly used in engineering, particularly in civil, mechanical, architectural, aeronautical, and marine engineering. Examples of the shell structures in civil and architectural engineering are large-span roofs, liquid-retaining structures and water tanks, containment shells of nuclear power plants, and concrete arch domes. In mechanical engineering, shell forms are used in piping systems, turbine disks, and pressure vessels technology. Of all existing shell models, the circular cylindrical shell is the most widely used.

Free vibration occurs in the absence of external force, however is initiated by applying initial displacement and/or velocity conditions to the shell. Knowledge of the free vibration characteristics of the thin circular cylindrical shells is important both for understanding the fundamental shell behavior and for designing shell structures for industrial applications. Also, for acoustic and dynamic response calculations, complete information of free vibration characteristics of these structures is needed. The frequencies and mode shapes of the shells are important in the design of such structures.

Many shell theories and methods have been developed over the decades for the analyses of the shells. These shell theories originated from the works reported by Love $(1888,1944)$ and Flügge $(1960)$ and many more shell theories that neglect some of the terms from the Flügge equations have been proposed

*Authorfor Correspondence: E-mail: augupta2001@gmail.com. Tel.: +91-875-088-7209 
over the years. Among other shell theories are Timoshenko (1921) theory based on one-dimensional (1-D) beam theory, and two-dimensional (2-D) theories include Donnell (1933), Reissner (1941), Sanders (1959) etc. The other theories are based on Rayleigh-Ritz energy methods (Arnold and Warburton, 1949, 1953), closed-form solutions of the governing equations, and iterative solution approaches. Leissa (1973) carried out a comprehensive review and comparison of various shell theories.

Further, the free vibration analysis of the circular cylindrical shell was studied by several researchers using various theories available in the literature. The shells were analyzed for different boundary conditions such as clamped - clamped (C-C), clamped - free (C-F), simply-supported - simply-supported (S-S), simply-supported - clamped (S-C), free - free (F-F), and shear diaphragm-shear diaphragm (SD-SD).

Sharma and Johns (1972) and Sharma (1974) proposed an approximate method for calculating the natural frequencies of the fixed-free circular cylindrical shells. Soedel (1980) proposed formula for calculating the natural frequencies of the circular cylindrical shells for different boundary conditions. Moussaoui et al. (2000) studied the mode shapes and frequencies of thin elastic shells of infinite length. Xuebin (2006) calculated the free vibration frequencies of a thin circular cylindrical shell based on Flügge's shell theory equations for orthotropic materials. Zhang et al. (2001) and Xuebin (2008) used wave propagation approach for calculating the free vibration of cylindrical shells for different boundary conditions. Amabili and Païdoussis (2003) presented a comprehensive review of various linear and nonlinear shell models reported in the literature. Amabili (2008) and Kurylov and Amabili (2010) studied the nonlinear vibration of the circular cylindrical shells for different boundary conditions. Farshidianfar and Oliazadeh (2012) used semi-analytical approach for the free vibration analysis of the simply-supported circular cylindrical shells and compared the results with ten different shell theories. Recently, Lee and Kwak (2015) used Rayleigh-Ritz method for free vibration analysis of the circular cylindrical shell and compared the results with different shell theories.

Similarly, finite element method (FEM) was also used for free vibration of the circular cylindrical shell as reported by Zienkiewicz (1969), Ramamurti and
Pattabiraman (1976), Kant et al. (1994), Zhang et al. (2001), Chapelle and Bathe (2011), Oliazadeh et al. (2013) and Lee and Kwak (2015).

It is evident from the literature that, as the circular cylindrical shell vibrates in different modes, its analysis becomes intricate, especially analytical/ closed-form solutions may become intractable. Hence, the FEM may be relied on for conducting free vibration analysis of the circular cylindrical shells.

The major objectives of the present study are: (i) to calculate the modal frequencies of the circular cylindrical shells using the FE approach and compare the results with different shell theories published in the literature, (ii) to study the effect of different boundary conditions, such as clamped - clamped (CC), clamped-free (C-F), and simply-supported simply-supported (S-S) on the modal frequencies of the shells, (iii) to investigate the effects of different height or length to radius $(H / R)$ ratios, thickness to radius $(h / R)$ ratios, and boundary conditions on the mesh convergence, based on which a ratio of perimeter of circular cylindrical shell to mesh size along the circumference and the ratio of height to mesh size along the height can be estimated, and (iv) to study the effect of $H / R$ and $h / R$ ratios on the modal frequencies of the circular cylindrical shells.

\section{Mathematical Modeling}

Figure 1 shows the co-ordinate system used for the cylindrical shell. Figures 2(A) and 2(B) respectively show circumferential and axial vibration modes of the cylindrical shell. The cylindrical shell considered is having height (or length), $H$; radius, $R$; constant thickness, $h$; density, $\rho$; modulus of elasticity, $E$; and Poisson's ratio, $\nu$. The reference surface of the shell is taken at its middle surface where an orthogonal

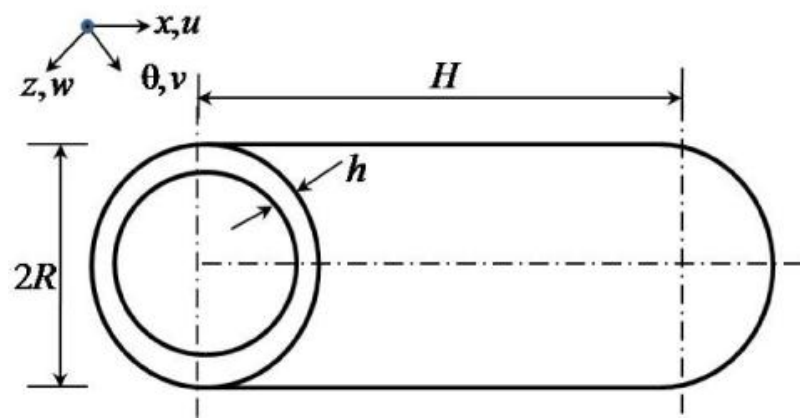

Fig. 1: Co-ordinate system of shell 
co-ordinate system $(x, \theta, z)$ is defined. The $x$ coordinate is taken in the axial direction of the shell, whereas the $\theta$ and $z$ co-ordinates are taken respectively in the circumferential and radial directions of the shell. The displacements of the shell are defined by $u, v$, and $w$ in the $x, \theta$, and $z$ directions, respectively. The circular cylindrical shell vibrates in axial mode, $m$ and the circumferential mode, $n$, whereas radial mode is ignorable. The circumferential modes are in-andout deformations in the form of cosine waves, $\cos (n \theta)$, as shown in Fig. 2(A), whereas the axial modes include flexural deformations along the axial direction as shown in Fig. 2(B). Any combination of $m$ and $n$ defines the modes and the corresponding modal frequencies of the circular cylindrical shell.

For the free vibration of a cylindrical shell, the equations of motion based on Flügge (1960) in matrix form are given as

$$
\left[\left.\begin{array}{lll}
L_{11} & L_{12} & L_{13} \\
L_{21} & L_{22} & L_{23} \\
L_{31} & L_{32} & L_{33}
\end{array}\right|_{w} ^{v}=\{0\}\right.
$$

where $L_{\mathrm{ij}}(i, j=1,2,3)$ are the differential operators with respect to $x, \theta$, and $t$. The first attempt at solving the Eq. (1) involves the assumption of a synchronous motion
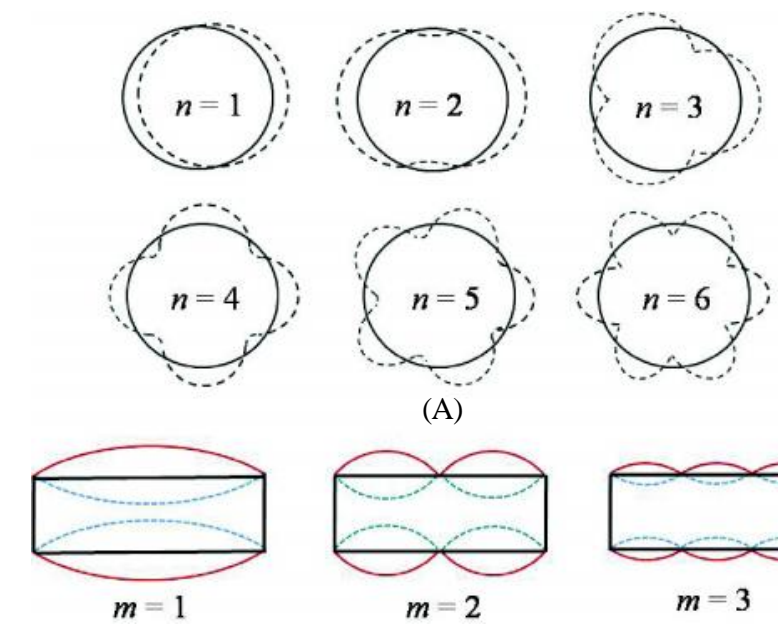

(A)
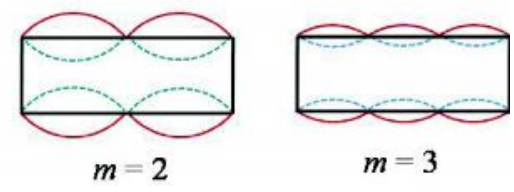

(B)

Fig. 2: (A) Circumferential and (B) axial modes of circular cylindrical shell

$$
\begin{aligned}
u(x, \theta, t) & =U(x, \theta) f(t) \\
v(x, \theta, t) & =V(x, \theta) f(t) \\
w(x, \theta, t) & =W(x, \theta) f(t)
\end{aligned}
$$

where $f(t)$ is the scalar model coordinate corresponding to the mode shapes $U(x, \theta), V(x, \theta)$, and $W(x, \theta)$. The next step is to use the separation of variables method in order to separate the spatial dependence of the mode shapes between axial and circumferential directions. Hence, the axial, tangential, and radial displacements of the shell wall are varied according to

$$
\begin{aligned}
& u(x, \theta, t)=A e^{\lambda_{\mathrm{m}} x} \sin (n \theta) \cos (\omega t) \\
& v(x, \theta, t)=B e^{\lambda_{\mathrm{m}} x} \sin (n \theta) \cos (\omega t) \\
& w(x, \theta, t)=C e^{\lambda_{\mathrm{m}} x} \sin (n \theta) \cos (\omega t)
\end{aligned}
$$

where $\lambda_{m}$ and $n$ are the axial wave number and the circumferential wave parameter, respectively, $A, B$, and $C$ are the undetermined constants, and ù is the angular/circular frequency of the natural vibration. Substitution of Eq. (3) into Eq. (1) and use of any of the theories given in the above-mentioned literature, lead to a set of homogeneous equations having the following matrix form

$$
\left[\begin{array}{lll}
C_{11} & C_{12} & C_{13} \\
C_{21} & C_{22} & C_{23} \\
C_{31} & C_{32} & C_{33}
\end{array} \mid \begin{array}{l}
U \\
V
\end{array}=\{0\}\right.
$$

in which $C_{\mathrm{ij}}(i, j=1,2,3)$ are coefficients which are the functions of $\lambda_{m}, n$, and a frequency parameter,

$$
\text { defined as } \quad{ }^{2}=\left(1-v^{2}\right) \rho \omega^{2} R^{2} / E \text {. For the non- }
$$

trivial solution, the determinant of the coefficient matrix must be zero, such as

$$
\operatorname{det}\left(\left[C_{\mathrm{ij}}\right]\right)=0, i, j=1,2,3
$$

The characteristics equation is obtained from the expansion of Eq. (4)

$$
f(\lambda, \omega)=0
$$

The boundary conditions for the clamped clamped (C-C) shell at $x=0$ and at $x=H$ are $u=v=$ 
$w=\partial w / \partial x=0$. And, boundary conditions for the clamped - free (C-F) shell at $x=0$ are $u=v=w=$ $\partial w / \partial x=0$, and at $x=H$ are $M_{\mathrm{x}}=N_{\mathrm{x}}=0$, where $M_{\mathrm{x}}$ is the axial moment and $N_{\mathrm{x}}$ is the axial force in the shell as it deforms. Moreover, for the simply-supported - simply-supported (S-S) shell, the boundary conditions at $x=0$ and at $x=H$ are $v=w=0$ and $M_{\mathrm{x}}=N_{\mathrm{x}}=0$.

The eigenvalue problem for the modal frequencies of an undamped FE model of the shell is given as

$$
\left(-\omega^{2} M^{\mathrm{MN}}+K^{\mathrm{MN}}\right) \Phi^{\mathrm{N}}=0
$$

where $M^{\mathrm{MN}}$ is the mass matrix (which is symmetric and positive definite), $K^{\mathrm{MN}}$ is the stiffness matrix (which includes initial stiffness effects if the base state included the effects of nonlinear geometry), $\Phi^{\mathrm{N}}$ is the eigenvector (the mode of vibration), and $M$ and $N$ are the degrees of freedom.

\section{Finite Element Modeling}

In the present study, finite element method (FEM) is used for conducting free vibration analysis of the circular cylindrical shell. Block Lanczos method is used for extracting frequencies of the circular cylindrical shell in the present study. The frequency extraction analysis is performed in the FE software
Abaqus $^{\circledR}$ using linear perturbation procedure. The cylindrical shell is modeled using four node quadrilateral shell elements (S4R) with reduced integration and hourglass control. The C-C, C-F, and $\mathrm{S}-\mathrm{S}$ boundary conditions are applied on both the circular edges of the shell.

The accuracy of the results obtained from the present FE models has been ensured for the circular cylindrical shell by comparing it with the results obtained from the past results reported in the literature. Comparison of the modal frequencies calculated using the present FE approach is made with that published by Lee and Kwak (2015). They presented modal frequencies obtained for the shells by adopting FE approach in ANSYS ${ }^{\circledR}$ and compared with the results obtained from the Flügge (FLH) and the DonnellMushtari (DM) theories. An aluminum shell with $H=$ $600 \mathrm{~mm}, R=150 \mathrm{~mm}, h=1 \mathrm{~mm}, \rho=2,770 \mathrm{~kg} / \mathrm{m}^{3}, E$ $=71 \mathrm{GPa}$, and $\nu=0.33$ is considered. Table 1 shows the modal frequencies $\left(f_{\mathrm{mn}}\right)$ obtained in the present study for the C-C and C-F boundary conditions of the shell and the results reported by Lee and Kwak (2015). The percentage difference between the modal frequencies obtained by the method adopted here, FLH, and DM theories is shown. It is observed that the modal frequencies obtained from the block Lanczos technique in the present FE approach are duly verified with the FLH theory.

Table 1: Comparison of modal frequencies in $\mathrm{Hz}$ between present FEM and results obtained by Lee and $\mathrm{Kwak}(2015)(H=600$ $\mathrm{mm}, R=150 \mathrm{~mm}, h=1 \mathrm{~mm})$

\begin{tabular}{|c|c|c|c|c|c|c|c|c|}
\hline \multirow[t]{2}{*}{ Boundary condition } & \multirow[t]{2}{*}{$\begin{array}{l}\text { Mode } \\
(m, n)\end{array}$} & \multirow{2}{*}{$\begin{array}{l}\text { Present } \\
\text { method } \\
\qquad f_{\mathrm{mn}}\end{array}$} & \multicolumn{2}{|c|}{$\begin{array}{l}\text { ANSYS }^{\circledR} \text { (Lee and } \\
\text { Kwak, 2015) }\end{array}$} & \multicolumn{2}{|c|}{$\begin{array}{c}\text { FLH (Lee and } \\
\text { Kwak, 2015) }\end{array}$} & \multicolumn{2}{|c|}{$\begin{array}{l}\text { DM (Lee and Kwak, } \\
\text { 2015) }\end{array}$} \\
\hline & & & $f_{\mathrm{mn}}$ & Diff. $(\%)$ & $f_{\mathrm{mn}}$ & Diff. (\%) & $f_{\mathrm{mn}}$ & Diff. (\%) \\
\hline \multirow[t]{6}{*}{ Clamped-Clamped } & $(1,5)$ & 371 & 370 & 0.3 & 371 & 0 & 379 & -2.1 \\
\hline & $(1,4)$ & 411 & 411 & 0 & 411 & 0 & 415 & -1 \\
\hline & $(1,6)$ & 429 & 427 & 0.5 & 429 & 0 & 439 & -2.3 \\
\hline & $(1,7)$ & 546 & 544 & 0.4 & 547 & -0.2 & 558 & -2.2 \\
\hline & $(1,3)$ & 586 & 586 & 0 & 587 & -0.2 & 588 & -0.3 \\
\hline & $(2,6)$ & 625 & 624 & 0.2 & 633 & -1.3 & 640 & -2.3 \\
\hline \multirow[t]{6}{*}{ Clamped-Free } & $(1,3)$ & 146 & 146 & 0 & 146 & 0 & 153 & -4.6 \\
\hline & $(1,4)$ & 175 & 174 & 0.6 & 175 & 0 & 185 & -5.4 \\
\hline & $(1,2)$ & 242 & 243 & -0.4 & 242 & 0 & 243 & -0.4 \\
\hline & $(1,5)$ & 263 & 261 & 0.8 & 263 & 0 & 274 & -4 \\
\hline & $(1,6)$ & 382 & 378 & 1.1 & 381 & 0.3 & 391 & -2.3 \\
\hline & $(2,5)$ & 381 & 380 & 0.3 & 381 & 0 & 389 & -2.1 \\
\hline
\end{tabular}




\section{Numerical Study and Discussion}

Free vibration analysis using FEM, carried out in the present study, aims to compare the performance of the thin circular cylindrical shell. The effects of different height or length to radius $(H / R)$ ratios, thickness to radius $(h / R)$ ratios, and boundary conditions on the mesh convergence in the FE analysis are investigated. Based on which a ratio of the perimeter of the circular cylindrical shell to mesh size along the circumference $\left(2 \pi R / s_{\mathrm{r}}\right)$ and a ratio of the height to mesh size along the height $\left(H / s_{\mathrm{H}}\right)$ are estimated. These two ratios along circumferential and longitudinal directions of the cylindrical shell give estimate on number of elements to subdivide the FE domain in the respective directions. The effect of different boundary conditions, such as clamped clamped (C-C), clamped - free (C-F), and simplysupported - simply-supported (S-S), height or length to radius $(H / R)$ ratios, and thickness to radius $(h / R)$ ratios on the modal frequencies of the shell are investigated.

The geometrical and material properties of the steel shell considered are: $R=1 \mathrm{~m}, \rho=7,850 \mathrm{~kg} / \mathrm{m}^{3}, E$ $=210 \mathrm{GPa}$, and $\nu=0.3$. Variations in the modal frequencies of the shell are studied for different height to radius $(H / R)$ ratios varying from 2 to 20 at an interval of 1 , and thickness to radius $(h / R)$ ratio varied as $1 / 200,1 / 100,1 / 50,1 / 20$ and $1 / 10$.

\section{Mesh Convergence Study}

In the numerical analysis using FE approach, coarse mesh is employed to commence with and subsequently mesh refinement studies are performed to obtain solution of problems within an acceptable accuracy. Therefore, it becomes necessary to determine a good initial mesh density for conversed results to obtain with reduced computational efforts. A set of mesh convergence study is performed for different $H / R$ ratios, based on which a ratio of perimeter of cylindrical shell $(2 \pi R)$ to mesh size along the circumference $\left(s_{\mathrm{r}}\right)$ and height or length $(H)$ to mesh size along the height $\left(s_{\mathrm{H}}\right)$ are proposed.

For this study, $R=1 \mathrm{~m}, 2 \mathrm{~m}$, and $3 \mathrm{~m} ; H / R=2$, 10,15 , and 20 are considered for all $h / R$ ratios and boundary conditions. The corresponding fundamental frequencies are evaluated using block Lanczos technique. The mesh size along the radius and height of the shell is varied and the converged value of fundamental frequency is considered for all the $H / R$ ratios.

Figure 3 shows the variation of $2 \pi R / s_{\mathrm{r}}$ ratio with respect to the $H / R$ ratio for the shell. It can be observed that as the $H / R$ ratio increases the required mesh size along the radius for convergence requirement decreases, which is independent of the boundary condition and $h / R$ ratio of the thin circular cylindrical shell. For higher $H / R$ ratio $=15$ and 20 , the fundamental mode number is $(1,2)$ whereas for $H / R$ ratio $=2$ it is $(1,5)$ for shell with $\mathrm{C}-\mathrm{C}$ boundary condition.
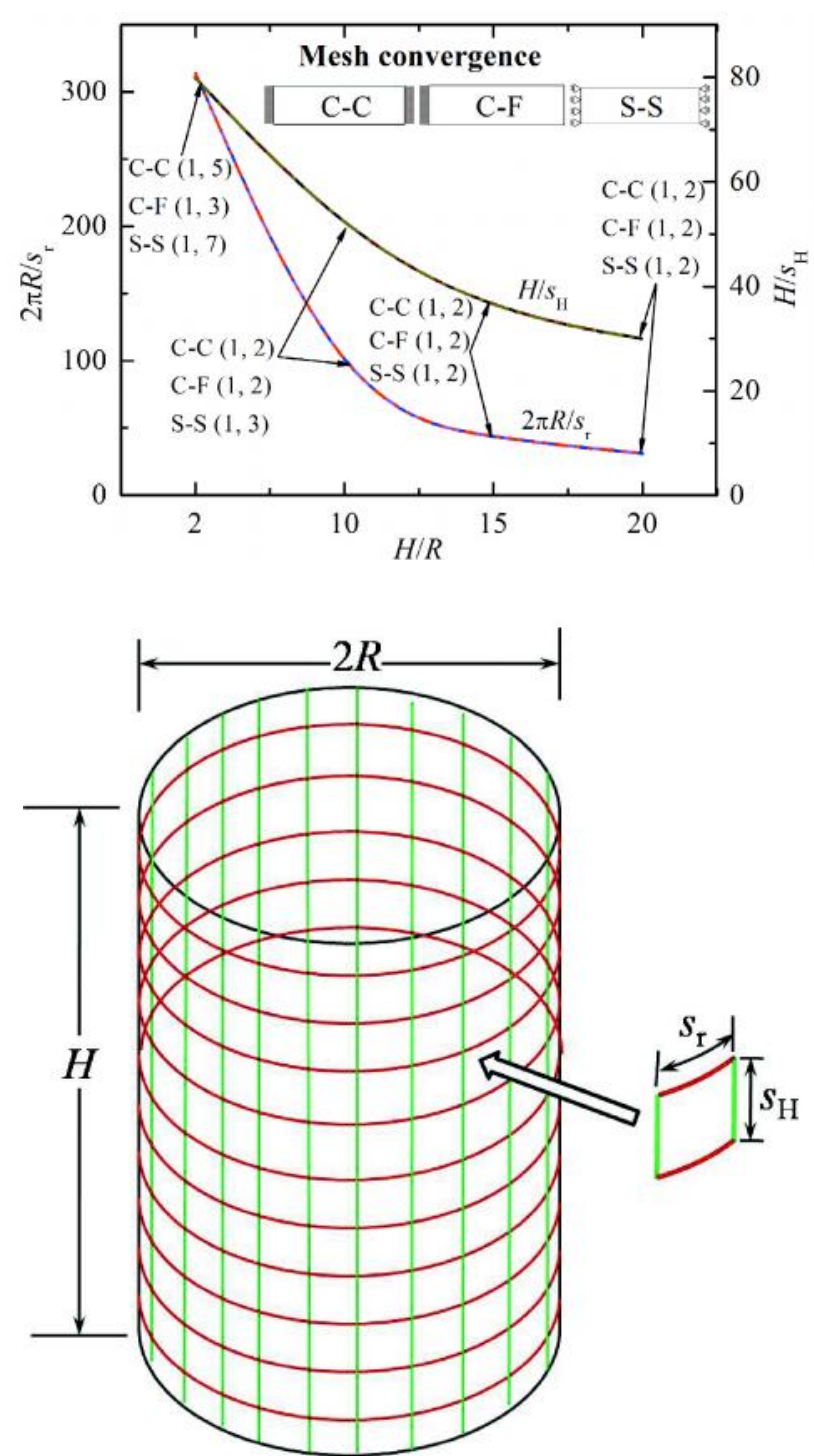

Fig. 3: Mesh convergence study of shell for $H / R$ ratios $=2$, 10,15 , and 20 
Figure 3 also shows the variation of the $H / s_{\mathrm{H}}$ ratio with respect to the $H / R$ ratio for the shell. This ratio decreases with increase in the $H / R$ ratio of the shell. The values of $H / s_{\mathrm{H}}$ ratio are much lesser than $2 \pi R / s_{\mathrm{r}}$ ratio, as more elements are required along the circumferential direction as compared to the longitudinal direction of the shell for lower $H / R$ ratio and vice-versa. This is attributed to the fact that, for slender thin circular cylindrical shell (higher $H / R$ ratio), finer FE mesh is required along the height (decreased $s_{\mathrm{H}}$ ) and relatively coarser mesh suffice along the circumference for convergence requirement, which is independent of the boundary condition and the shell thickness. The aspect ratio of the FE mesh $\left(s_{\mathrm{r}} / s_{\mathrm{H}}\right)$ for cylindrical shell can be estimated based on the present mesh convergence study. The mesh convergence study gives an estimate for selecting the mesh size for the FE analysis of the circular cylindrical shell. This avoids the rigorous mesh convergence study to start FE computations and hence reduces the computational efforts.

\section{Geometric and Boundary Conditions Study}

The effects of different boundary conditions, $H / R$, and $h / R$ ratios on the modal frequencies of the shell are investigated. Table 2 shows the first (lowest) ten modal frequencies for $H / R=2$ for C-C, C-F, and S-S boundary conditions of the shells for all $h / R$ ratios considered herein. It can be observed that maximum frequency for $H / R=2$ corresponds to $h / R=1 / 10$, for the increased thickness of the shell.

Figures 4(A), 4(B), and 4(C) show the modal frequencies for the shell with $\mathrm{C}-\mathrm{C}$ boundary condition considering the first axial mode $(m=1), H / R=2,10$, and 20 , and for all considered $h / R$ ratios, respectively. It can be seen that with increase in the circumferential mode $(n)$ for $m=1$, the modal frequency, $f_{\mathrm{mn}}$ increases for higher $H / R$ ratios (10 and 20) however not for the $H / R=2$ for all considered $h / R$ ratios. As the $h / R$ ratio increases the modal frequencies increase in the circumferential mode.

Figure 4(D) shows the frequency for different circumferential modes with $m=1$ and for different $H / R$ ratios considering $h / R=1 / 10$ with the C-C boundary condition of the shell. It is seen that for all the $n$ values up to $H / R=10$ there is decrease in the frequency with increase in the $H / R$ ratio. Beyond that, it remains almost constant for larger $H / R$ ratios. For
Table 2: Modal frequencies in $\mathrm{Hz}$ in cylindrical shell $(m=$ 1) for $H / R=2$

\begin{tabular}{|c|c|c|c|c|c|}
\hline$n$ & $h / R=1 / 200$ & $h / R=1 / 100$ & $h / R=1 / 50$ & $h / R=1 / 20$ & $h / R=1 / 10$ \\
\hline \multicolumn{6}{|c|}{$\mathrm{C}-\mathrm{C}$} \\
\hline 1 & 475 & 504 & 506 & 511 & 523 \\
\hline 2 & 325 & 326 & 329 & 342 & 375 \\
\hline 3 & 224 & 226 & 233 & 269 & 358 \\
\hline 4 & 163 & 169 & 187 & 277 & 462 \\
\hline 5 & 126 & 139 & 181 & 352 & 647 \\
\hline 6 & 105 & 133 & 211 & 473 & 886 \\
\hline 7 & 98 & 148 & 265 & 626 & 1168 \\
\hline 8 & 102 & 176 & 336 & 807 & 1486 \\
\hline 9 & 116 & 216 & 421 & 1012 & 1836 \\
\hline 10 & 136 & 263 & 518 & 1241 & 2214 \\
\hline \multicolumn{6}{|c|}{$\mathrm{C}-\mathrm{F}$} \\
\hline 1 & 237 & 238 & 238 & 240 & 242 \\
\hline 2 & 119 & 119 & 120 & 127 & 144 \\
\hline 3 & 66 & 69 & 77 & 120 & 209 \\
\hline 4 & 44 & 55 & 86 & 191 & 366 \\
\hline 5 & 40 & 66 & 123 & 298 & 574 \\
\hline 6 & 48 & 90 & 176 & 432 & 823 \\
\hline 7 & 62 & 122 & 242 & 591 & 1110 \\
\hline 8 & 81 & 160 & 318 & 773 & 1431 \\
\hline 9 & 102 & 204 & 405 & 980 & 1782 \\
\hline 10 & 127 & 253 & 503 & 1209 & 2162 \\
\hline \multicolumn{6}{|c|}{ S-S } \\
\hline 1 & 503 & 503 & 504 & 504 & 506 \\
\hline 2 & 324 & 324 & 325 & 330 & 346 \\
\hline 3 & 223 & 224 & 228 & 254 & 328 \\
\hline 4 & 162 & 166 & 182 & 264 & 437 \\
\hline 5 & 125 & 137 & 177 & 342 & 629 \\
\hline 6 & 104 & 132 & 208 & 465 & 872 \\
\hline 7 & 100 & 146 & 262 & 620 & 1157 \\
\hline 8 & 102 & 175 & 334 & 801 & 1477 \\
\hline 9 & 116 & 215 & 419 & 1007 & 1828 \\
\hline 10 & 136 & 262 & 516 & 1237 & 2208 \\
\hline
\end{tabular}

the first three wave numbers $(n=1,2$, and 3$)$ the variation in the modal frequencies is more with respect to the $H / R$ ratio.

Figure 4(E) shows the variation of the modal frequencies with respect to the $h / R$ ratio for $H / R=2$ and 10 with $(m, n)=(1,3)$ and $(1,7)$. It is seen that with increase in the $h / R$ ratio, the frequency increases 


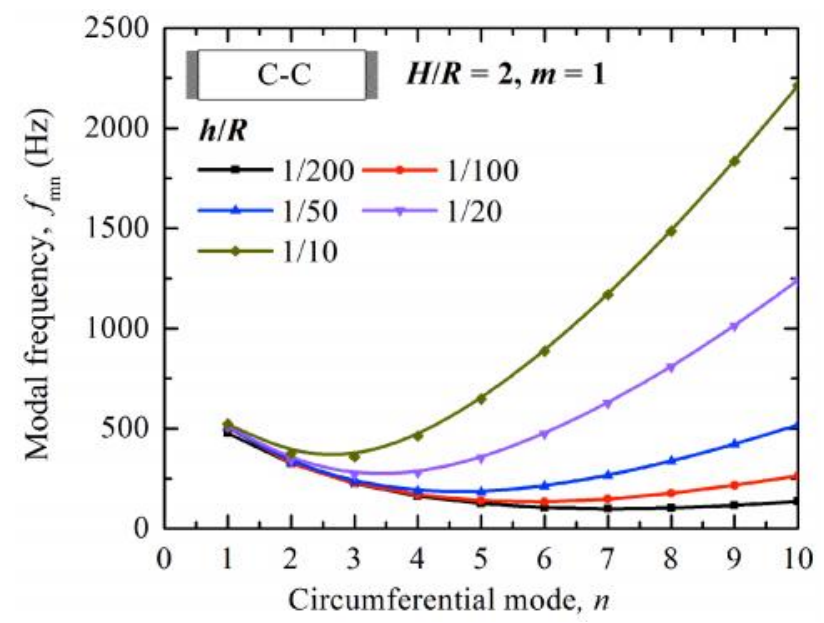

A

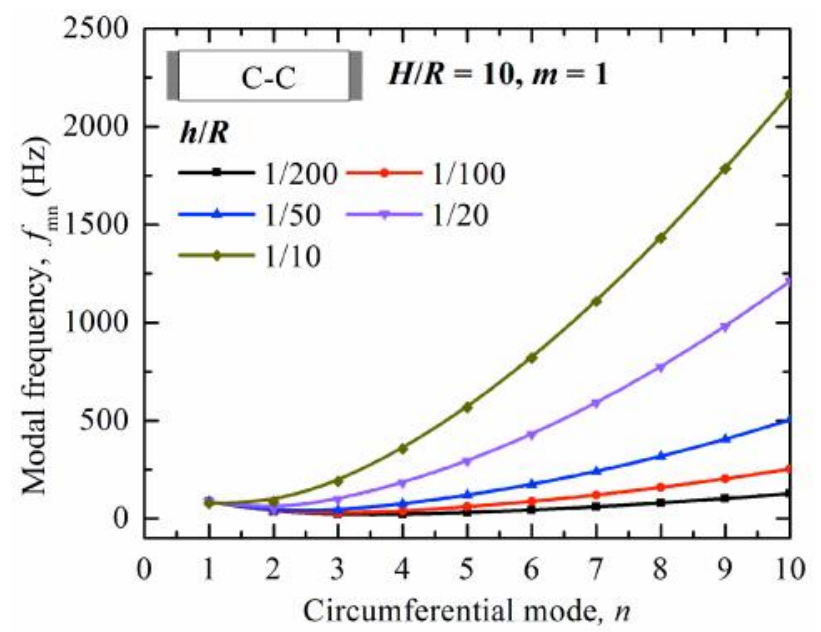

B

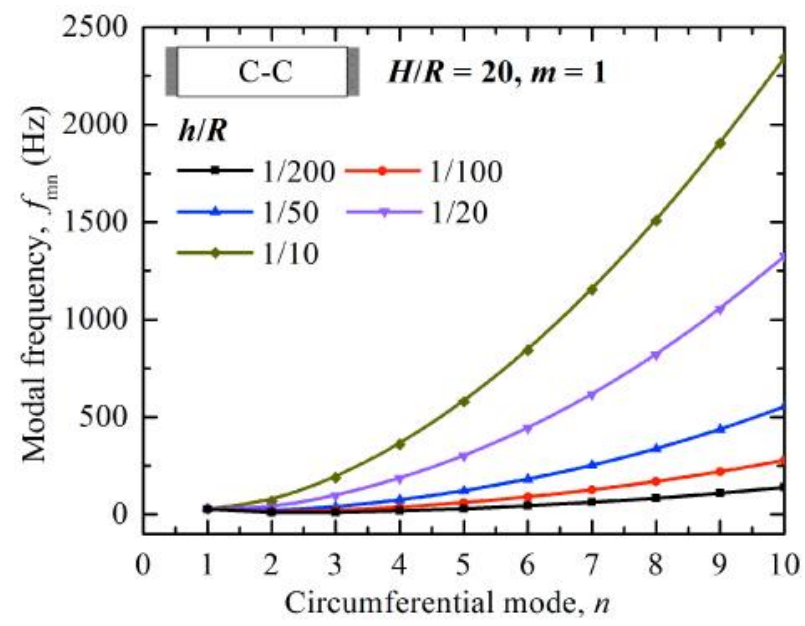

C

in circumferential mode. For higher wave number there is no significant effect of the $H / R$ ratio on the frequency values with respect to the $h / R$ ratios.

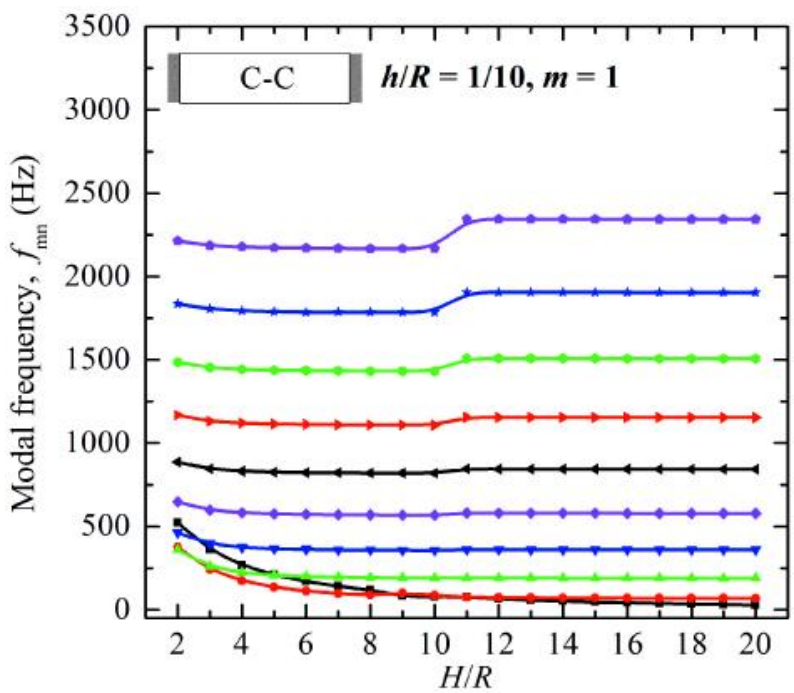

$n$ (circumferential mode)

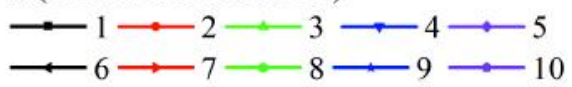

D

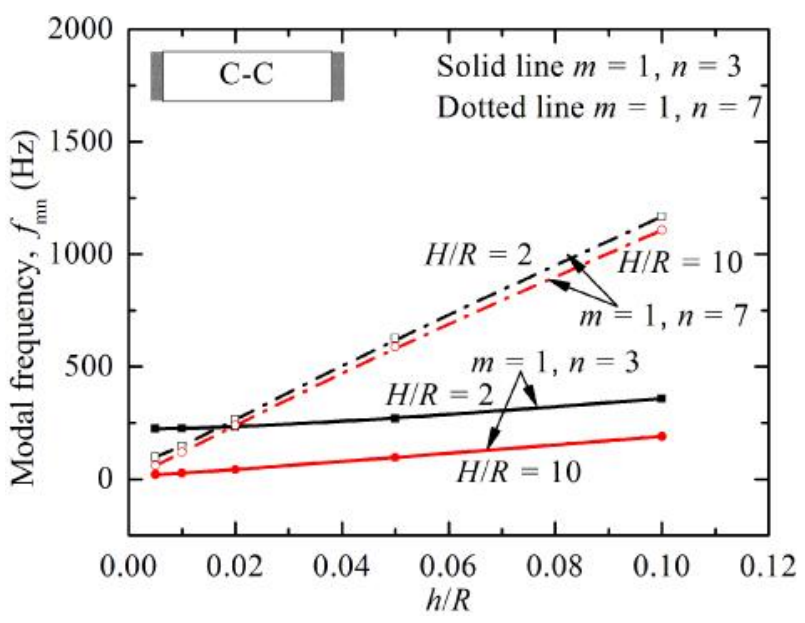

$\mathbf{E}$

Fig. 4: Modal frequencies for C-C shell $(m=1)$, (A) For different $h / R$ ratios, $H / R=2$, (B) For different $h / R$ ratios, $H / R=10$, (C) For different $h / R$ ratios, $H / R=20$, (D) For different $H / R$ ratios for $h / R=1 / 10$ and (E) For different $h / R$ ratios for $H / R=2$ and $10(1,3),(1,7)$

In case of the shell with C-F boundary condition, one end is clamped due to which the modal frequencies are reduced. Nevertheless, trend of the results similar 


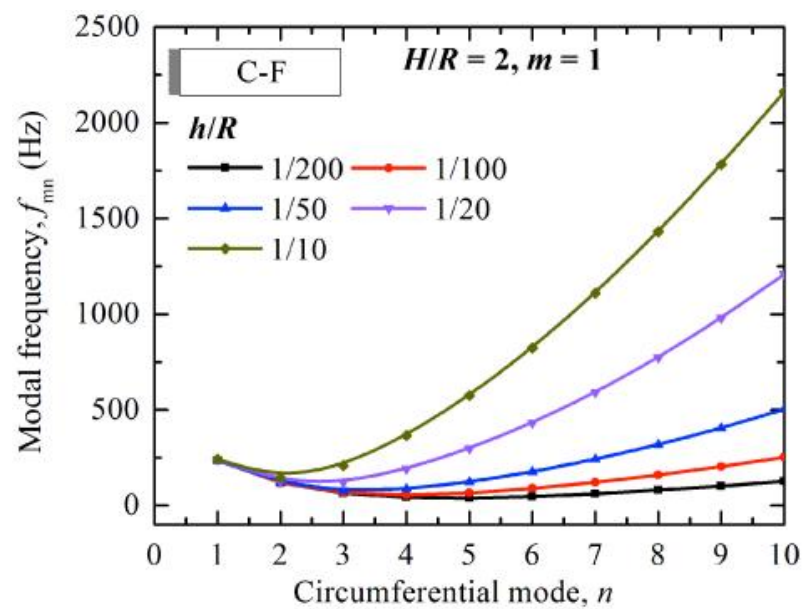

A

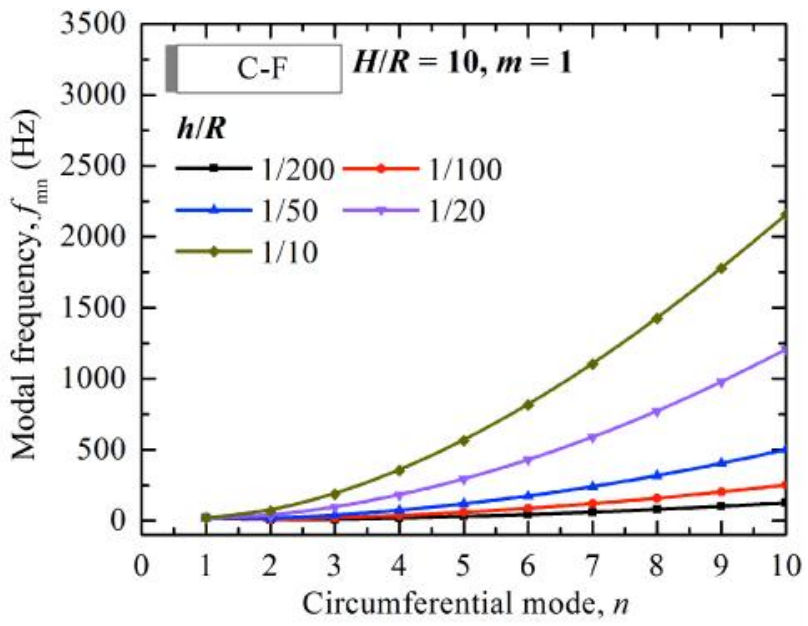

B

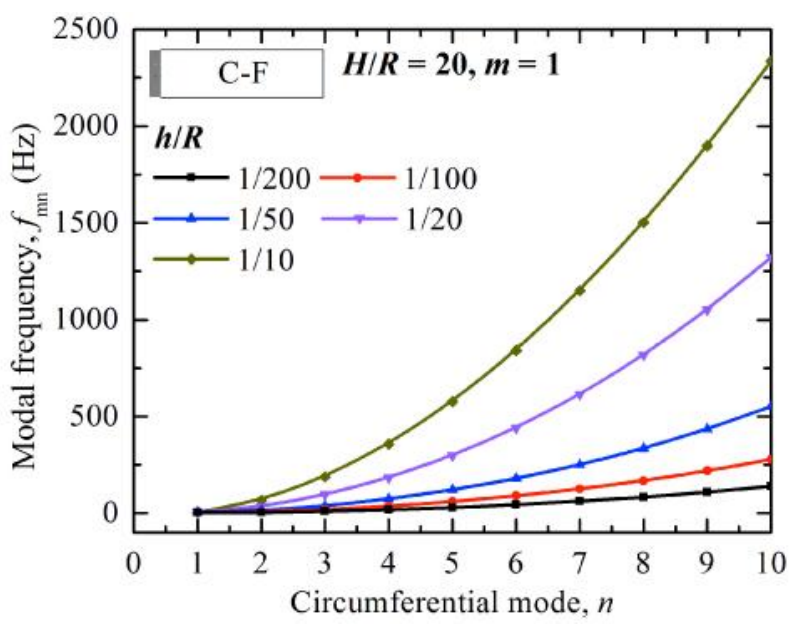

C

to the C-C boundary condition are observed in case of the C-F boundary condition, which can be seen from Figs. 5(A), 5(B), 5(C), 5(D) and 5(E).

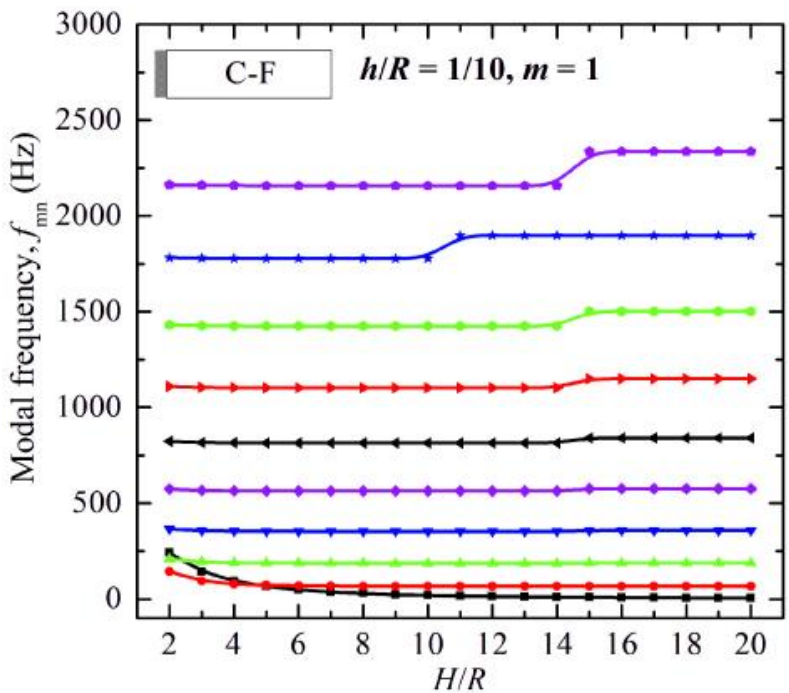

$n$ (circumferential mode)

$\longrightarrow 1 \longrightarrow 2 \longrightarrow 3 \longrightarrow 4 \longrightarrow 5$
$-6 \rightarrow-7 \longrightarrow 8 \longrightarrow 9$

D

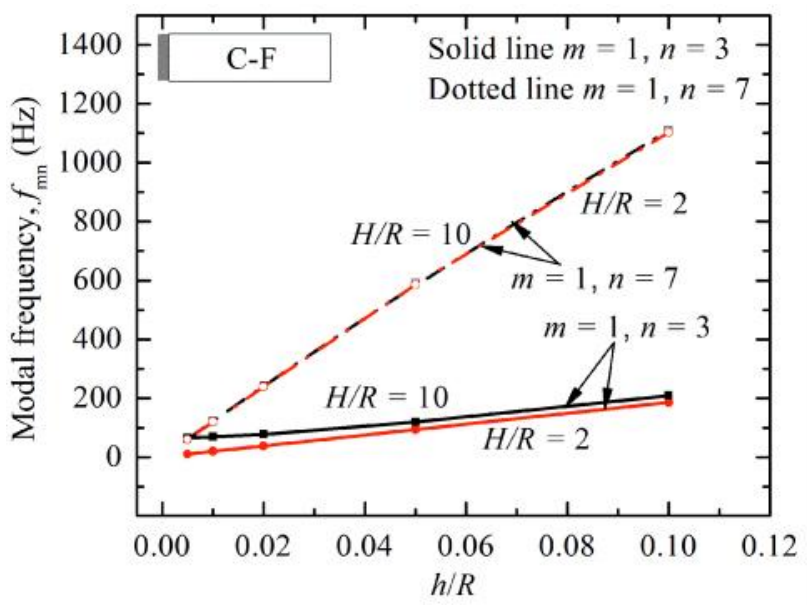

$\mathbf{E}$

Fig. 5: Modal frequencies for C-F shell $(m=1)$, (A) For different $h / R$ ratios, $H / R=2$, (B) For different $h / R$ ratios, $H / R=10$, (C) For different $h / R$ ratios, $H / R=20$, (D) For different $H / R$ ratios for $h / R=1 / 10$ and (E) For different $h / R$ ratios for $H / R=2$ and $10(1,3),(1,7)$

Figure 6 shows the comparison of modal frequencies with $m=1$ and $n=1$ to 10 with $\mathrm{C}-\mathrm{C}$ and C-F shell boundary conditions considering $H / R=2$ 

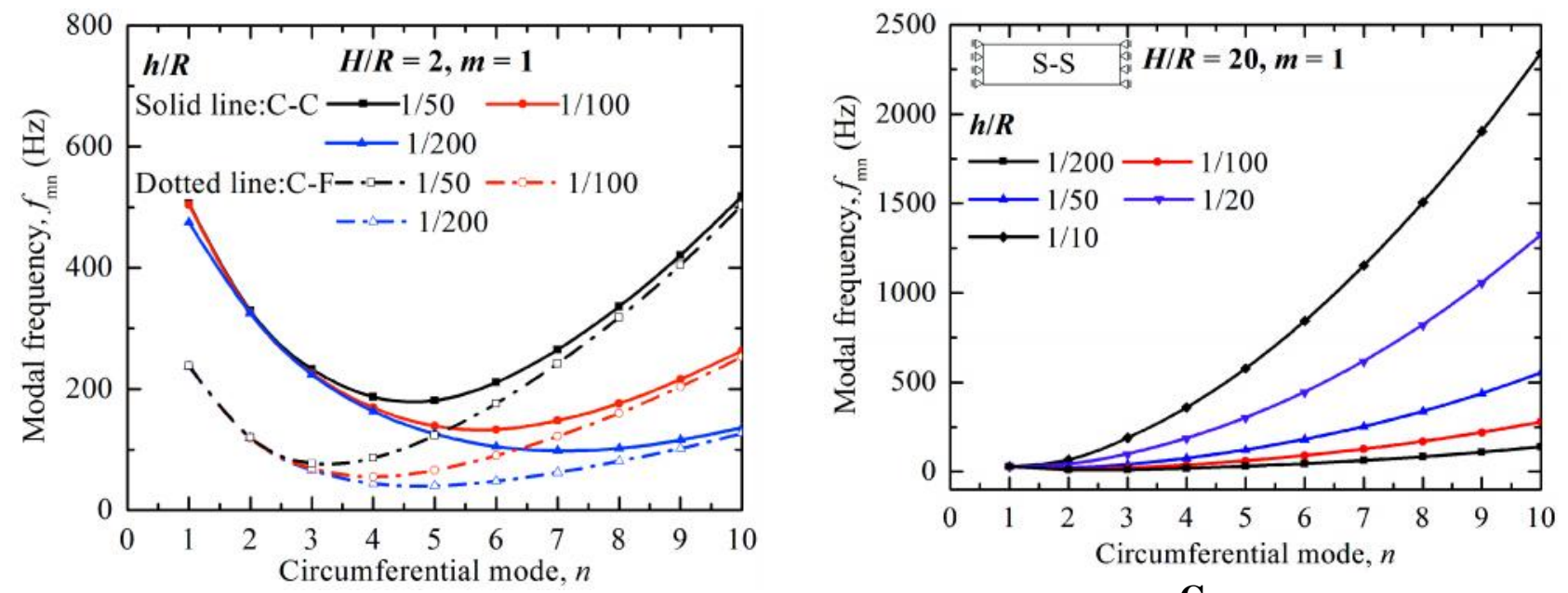

Fig. 6: Comparison of modal frequencies for $\mathrm{C}-\mathrm{C}$ and $\mathrm{C}-\mathrm{F}$ shells considering $H / R=2$, and for $h / R=1 / 50,1 / 100$, and $1 / 200$

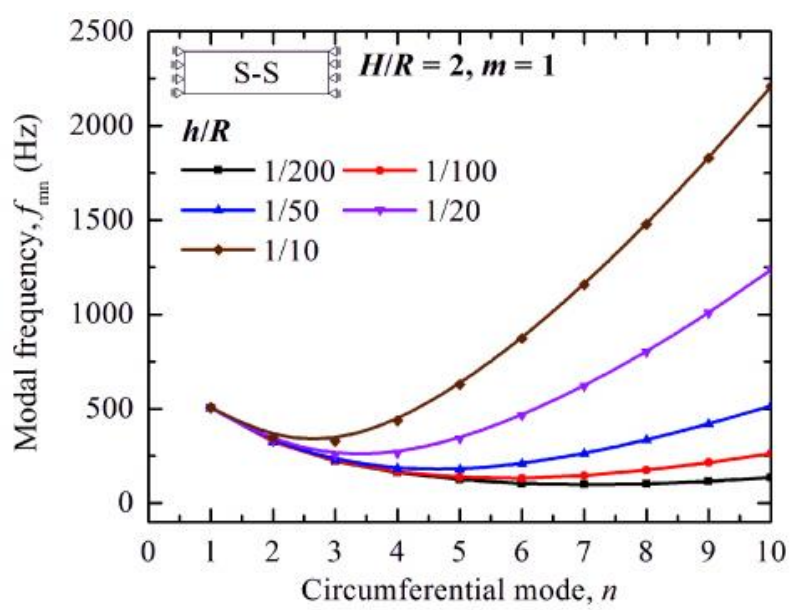

A

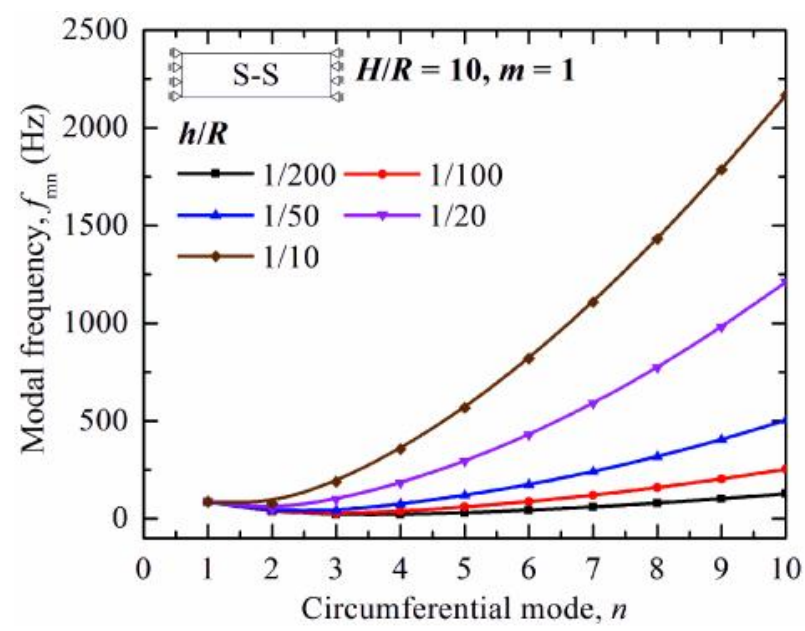

B

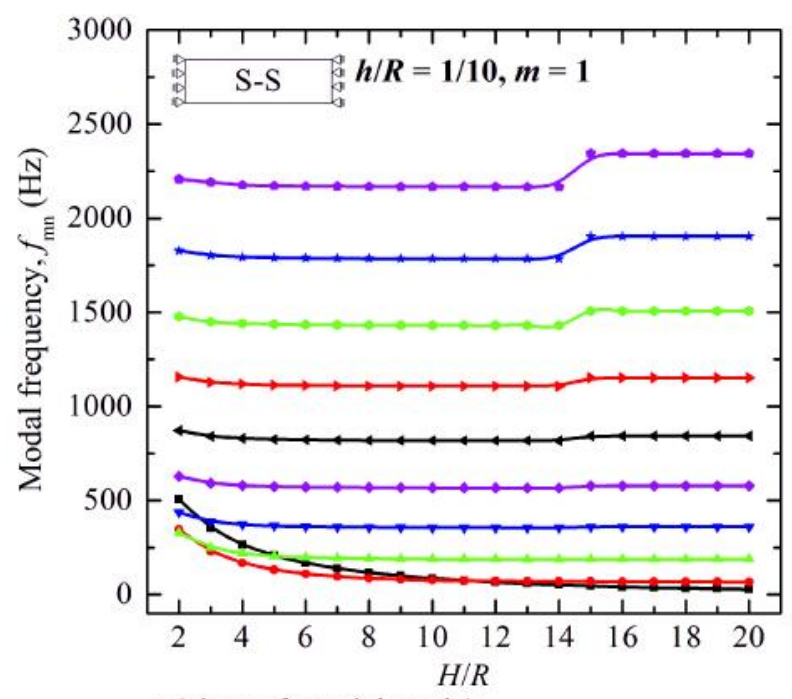

$n$ (circumferential mode)

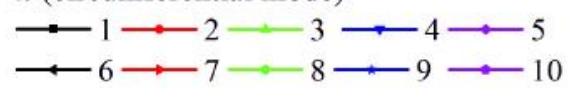

D

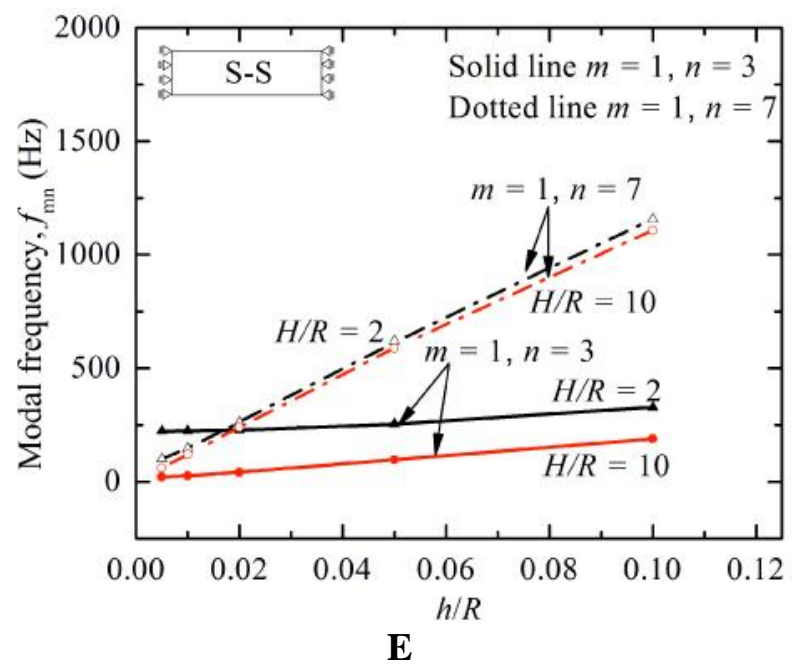

Fig. 7: Modal frequencies for S-S shell $(m=1)$, (A) For different $h / R$ ratios, $H / R=2$, (B) For different $h / R$ ratios, $H / R=10,(C)$ For different $h / R$ ratios, $H / R=20$, (D) For different $H / R$ ratios for $h / R=1 / 10$, (E) For different $h / R$ ratios for $H / R=2$ and 10 $(1,3),(1,7)$ 
and $h / R=1 / 50,1 / 100$, and 1/200. Here, the first three modal frequencies are same irrespective of the $h / R$ ratio seen for both C-C and C-F boundary conditions of the shells. The modal frequency $(1,1)$ for $H / R=2$ considering the shell with $\mathrm{C}-\mathrm{C}$ boundary condition is $475 \mathrm{~Hz}$ while in case of the shell with C-F boundary condition it is $237 \mathrm{~Hz}$ corresponding to $h / R=1 / 200$. The modal frequencies are lower in case of the C-F boundary condition as compared to the $\mathrm{C}-\mathrm{C}$ condition. Notably, the effect of boundary conditions on the modal frequencies diminishes at higher mode, $n$.

Figures 7(A), 7(B) and 7(C) show the modal frequencies for the shell with S-S boundary condition considering $m=1$ (first axial mode only), $H / R$ ratio 2 , 10 , and 20 , respectively and for all considered $h / R$ ratios. It can be seen that with increase in the circumferential mode $(n)$ for $m=1$, the $f_{\mathrm{mn}}$ increases for higher $H / R$ ratios (10 and 20) however not for $H /$ $R=2$. The circumferential frequencies increase with the increase in the wave number; however, they are insignificantly affected by the increase in the $H / R$ ratio from 10 to 20 as seen from Fig. 7(D). For the first three wave numbers the variation in the modal frequencies is more with respect to the $H / R$ ratio than higher $n$.

Figure $7(E)$ shows the variation of the modal frequencies with respect to the $h / R$ ratio for $H / R=2$ and 10 with $(m, n)=(1,3)$ and $(1,7)$. It is seen that for higher circumferential mode, $n=7$ the modal frequency with varying $h / R$ ratio for $H / R=2$ and 10 is almost same.

As the height to radius $(H / R)$ ratio of the circular cylindrical shell decreases, it becomes more rigid and its modal frequencies increase. The broad shell (lower $H / R$ ratio) behaves more rigidly in longitudinal direction

\section{References}

Abaqus/Standard User's Manual Version 6.11 (2011) Dassault Systemes Simulia Corporation Rhode Island (RI) USA

Amabili M and Païdoussis M P (2003) Review of studies on geometrically nonlinear vibrations and dynamics of circular cylindrical shells and panels, with and without fluidstructure interaction Applied Mechanics Reviews 56349 381

Amabili M (2008) Nonlinear vibrations and stability of shells thereby its longitudinal frequencies $(m)$ decrease and circumferential frequencies $(n)$ increase.

\section{Conclusions}

The free vibration finite element (FE) analysis of thin circular cylindrical shells is conducted. As the circular cylindrical shell vibrates in different modes, its analysis becomes intricate, especially analytical/closed-form solutions may become intractable. Hence, the FE method may be relied on for conducting free vibration analysis of the circular cylindrical shells. A set of mesh convergence studies are performed for the selecting the appropriate FE mesh size criteria which is based on the perimeter and height of the circular cylindrical shell, thickness to radius $(h / R)$ ratios, and various boundary conditions. From the results of the present study, the following conclusions are derived:

1. For slender thin circular cylindrical shell (higher $H / R$ ratio), finer FE mesh is required along the height (decreased $s_{\mathrm{H}}$ ) and relatively coarser mesh suffice along the circumference for convergence requirement, which is independent of the boundary condition and the shell thickness.

2. The modal frequency of the circular cylindrical shell is influenced mainly by the boundary conditions, $H / R$, and $h / R$ ratios. The modal frequency decreases for the C-F boundary condition as compared to the C-C and S-S boundary conditions, and it also decreases with decreased shell thickness (lower $h / R$ ratio).

3. The broad shell (lower $H / R$ ratio) behaves more rigidly in longitudinal direction thereby its longitudinal frequencies $(m)$ decrease and circumferential frequencies $(n)$ increase.

and plates Cambridge University Press New York (NY) USA

Arnold R N and Warburton G B (1949) Flexural vibrations of the walls of thin cylindrical shells having freely supported ends Proceedings of the Royal Society of London Series A Mathematical and Physical Science 197 238-256

Arnold R N and Warburton G B (1953) The flexural vibrations of thin cylinders Proceedings of the Institution of Mechanical Engineers 167 62-80 
Chapelle D and Bathe K J (2011) The finite element analysis of shells - fundamentals Springer New York (NY) USA

Donnell L H (1933) Stability of thin walled tubes under torsion NACA Report No. 479 New York (NY) USA

Farshidianfar A and Oliazadeh P (2012) Free vibration analysis of circular cylindrical shells: comparison of different shell theories International Journal of Mechanics and Applications 2 74-80

Flügge W (1960) Stresses in shells Springer New York (NY) USA

Kant T, Kumar S and Singh U P (1994) Shell dynamics with three-dimensional degenerate finite elements Computers and Structures 50 135-146

Kurylov Y and Amabili M (2010) Polynomial versus trigonometric expansions for nonlinear vibrations of circular cylindrical shells with different boundary conditions Journal of Sound and Vibration 329 1435-1449

Lee H and Kwak M K (2015) Free vibration analysis of circular cylindrical shell using the Rayleigh-Ritz method and comparison of different shell theories Journal of Sound and Vibration 353 344-377

Leissa A W (1973) Vibration of shell NASA SP-288 U.S. Government Printing Office Washington DC USA

Love AE H (1888) The small free vibrations and deformation of thin elastic shell Philosophical Transactions of the Royal Society at London 179A 491-546

Love A E H (1944) A treatise on the mathematical theory of elasticity Fourth Edition Dover Publications New York (NY) USA

Moussaoui F, Benamar R and White R G (2000) The effects of large vibration amplitudes on the mode shapes and natural frequencies of thin elastic shells Part I: coupled transversecircumferential mode shapes of isotropic circular cylindrical shells of infinite length Journal of Sound and Vibration 232 917-943

Oliazadeh P, Farshidianfar M H and Farshidianfar A (2013) Exact analysis of resonance frequency and mode shapes of isotropic and laminated composite cylindrical shells Part I: analytical studies Journal of Mechanical Science and Technology 27 3635-3643

Ramamurti V and Pattabiraman J (1976) Free vibrations of circular cylindrical shells Journal of Sound and Vibration 48 137155

Reissner E (1941) A new derivation of the equations of the deformation of elastic shells American Journal of Mathematics 63 177-184

Sanders J L (1959) An improved first approximation theory for thin shells (NASA TR-R24) U.S. Government Printing Office Washington DC USA

Sharma C B and Johns D J (1972) Free vibration of cantilever circular cylindrical shells - a comparative study Journal of Sound and Vibration 25 433-449

Sharma C B (1974) Calculation of natural frequencies of fixedfree circular cylindrical shells Journal of Sound and Vibration 35 55-76

Soedel W (1980) A new frequency formula for closed circular cylindrical shells for a large variety of boundary conditions Journal of Sound and Vibration 70 309-317

Timoshenko S P (1921) On the correction for shear of the differential equation for transverse vibrations of prismatic bars Philosophical Magazine LXVI 41 744-746

Xuebin L (2006) A new approach for free vibration analysis of thin circular cylindrical shell Journal of Sound and Vibration 296 91-98

Xuebin L (2008) Study on free vibration analysis of circular cylindrical shells using wave propagation Journal of Sound and Vibration 311 667-682

Zhang X M, Liu G R and Lam K Y (2001) Vibration analysis of thin cylindrical shells using wave propagation approach Journal of Sound and Vibration 239 397-403

Zienkiewicz O C (1969) The finite element method in engineering science McGraw-Hill Book Company London UK. 